\title{
OSTEREIERSPIELE IN DEUTSCHLAND
}

\author{
B. SCHMELZ \\ Museum für Völkerkunde \\ D-20148 Hamburg, Binderstr. 14, BRD
}

Die gemeinschaftliche Gestaltung des Osterfestes ist mit ihren vielfältigen regionalen, lokalen und auch individuellen Varianten ein bedeutender Ausdruck einer sehr intensiven Volksreligiosität. Diese hat sich im Laufe der Geschichte nicht nur von den Inhalten, sondern auch von der Bedeutung her mehrfach gewandelt. In besonderem Maße gilt das in der Gegenwart, wenn man nur an die stark zugenommenen Säkularisierungstendenzen denkt. Das Osterei hat gerade im deutschsprachigen Raum als ein wichtiges Element dieses religiösen Festes eine alte und sehr bedeutende Tradition. Es hat die Menschen immer wieder zu viel Phantasie sowie handwerklichen und künstlerischen Höchstleistungen angeregt. Aber auch der spielerische Umgang mit dem Osterei hat in Vergangenheit und Gegenwart viele Bräuche entstehen lassen.

\section{ANMERKUNGEN ZUR GESCHICHTE DER OSTEREIERSPIELE}

Der Volkskundler Hans MOSER erarbeitete in einem 1957 erschienenen Artikel sehr gründlich die Geschichte von Eierspielen im bayrischen Raum, der bis zur Gegenwart zu einer der bedeutendsten Regionen der Ostereierspiele in Deutschland zählt. Als den frühesten Beleg führt MOSER einen Hinweis im „Wändelregister der Amtsrechung“ des niederbayrischen Gerichtes Teisbach vom Jahr 1532 an. Ausführliche Beschreibungen solcher Spiele finden sich dann allerdings erst in späteren Zeiten. So konnte Moser für das oberfränkische Naila das sog. „Eierwalgen“ für das Jahr 1864 zitieren:

„Das Walgen besteht darin, daß einer von den Teilnehmern am Rande eines Abhangs sein Fuß anlegt, so viele Teilnehmer, etwa drei bis acht, bei einem solchen Akt zugegen sind, so viele Eier werden hinter den horizontal gestellten Fuß gelagert. Hierauf zieht der erstere den Fuß hinweg und die Eier, welche durch die Farbe und Größe sich unterscheiden, rollen den Abhang hinab. Wessen Ei am weitesten rollt, hat die anderen gewonnen".

${ }^{1}$ MOSER (1957: 81) 
Eine weitere Spielart wurde nach MOSER in der gleichen Gegend wohl noch bis in die 50er Jahre des 20. Jahrhunderts praktiziert:

„Auf einer Hangwiese stellt sich in früher Nachmittagsstunde alt und jung ein. Acht, zehn Walcher legten je ein Ei in eine Linie. Ein Unparteiischer gab das Startzeichen und nun rollten die Eier den Berg hinab. Wessen Ei zuerst unten ankam, war Sieger und hatte die anderen gewonnen“.2

Der Mathematiker und Arzt Georgius Henischius berichtete 1609 in seinem Werk „Arithmetica perfecta et demonstrata“ nicht nur von einem schwäbischen Eierlauf, sondern entwickelte daraus auch noch eine Rechenaufgabe. An Ostern sei es in Augsburg üblich, daß zwei Jungen einen Wettbewerb durchführen. Der eine muß 100 Eier, die auf dem Boden der Länge nach ausgelegt werden, auflesen und unzerbrochen in einen Korb legen. Der andere muß in der Zwischenzeit einen Lauf über eine bestimmte Strecke zurücklegen. Wer zuerst seine Aufgabe beendet hat, ist Sieger. ${ }^{3}$ Dieses berühmte „Eiersammeln“ bzw. „-laufen“, für das es aus dem 17. Jahrhundert reichlich Belege gibt, hat sich in einigen Regionen Deutschlands bis zur Gegenwart erhalten - ich werde darauf später noch zurückkommen.

Eine der bis heute einfachsten Formen der Spiele, das Zusammenstoßen mit zwei Eiern, weist MOSER besonders für das 19. Jahrhundert nach - damals unter der Bezeichnung „Eierspecken“ oder „Eierditschen“. Es war ein Spiel, das Jungen nur zur Osterzeit durchführten. Sieger war, wessen Ei unbeschädigt blieb - dieser gewann das unterlegene Ei. MOSER verwies darauf, daß dieses Spiel ein altes und ernstes Vorbild im Kirchenbrauch habe. Das Anstoßen mit geweihten Eiern sei im Umfeld der byzantinischen Kirche bereits für den Anfang des 13. Jahrhunderts bezeugt. ${ }^{4}$

\section{EIN TRADITIONSREICHES SPIEL: DAS EIERSAMMELN UND LAUFEN}

In früheren Zeiten wurden Ostereierspiele nicht in erster Linie von Kindern, sondern vielmehr besonders von jungen Erwachsenen durchgeführt. In vielen Orten Bayerns, Hessens, Badens, aber auch in Westfalen, im Elsaß, in Tirol, in der Schweiz und in Österreich war das bereits oben erwähnte Eiersammeln bzw. -laufen bis weit ins 20. Jahrhundert hinein ein beliebtes Spiel. ${ }^{5}$ Die Teilnehmer gehörten zumeist bestimmten festen Gruppen, wie Zünften oder Vereinen an. Der Wettkampf zwischen zwei Personen oder zwei Gruppierungen entwickelte viele regionale und lokale Va-

\footnotetext{
2 Moser (1957: 81)

${ }^{3}$ Nach MOSER (1957: 82)

${ }^{4}$ MOSER (1957: 83)

${ }^{5}$ Vgl. BLOBEL (1980: 86); KRUHÖFFER-MieLKE (1981: 26); DiETZE (1987: 69).
} 
riationen. Dieses Ostereierspiel hat sich mit seiner alten Tradition in einigen Orten Deutschlands bis heute als ein großes Spektakel erhalten.

Im unterfränkischen Remlingen, im heutigen Landkreis Main-Spessart, soll es der Überlieferung nach um 1650 durch die Grafen von Castell eingeführt worden sein, als sie bei der Ablieferung des Eierzinses zu Ostern einen Teil davon den einheimischen Jungen zum Spielen überließen. ${ }^{6}$ Über die frühe Geschichte des Spieles in Remlingen ist mir bisher leider nichts bekannt, aber offensichtlich wurden im Jahre 1838 der Brauch bewußt erneuert. In einem Dokument wurde er als „uralte, auf die vor den Franken hier seßhaften Germanen zurückführende Sitte" bezeichnet. Hans MOSER sah darin den damaligen Zeitgeist, der darauf bedacht war, das Gemeinschaftsgefühl zu fördern, indem Nationalfeste und erzieherisch nützliche Jugendfeste eingeführt wurden. So sollten die 1838 herausgegebenen Anordnungen dem Brauch „eine edle Gestalt und sinnige Richtung“ geben und der Jugend „zur Aufmunterung zum Guten und einen fleißigen moralischen, sittlichen Lebenswandel sowohl als zur Verbreitung nützlicher Lesebücher" verhelfen. So wurde konsequenterweise festgelegt, daß nur „Unbescholtenen“ die Teilnahme an dem Spiel erlaubt werden sollte. ${ }^{7}$

Auch heute wird das Spiel in Remlingen noch unter großem Zulauf der Bevölkerung am Ostermontag durchgeführt. ${ }^{8} 1997$ zogen die „unbescholtenen“ Burschen des Dorfes mit geflochtenen Birkenruten, den sog. „Eierlaufruten“, die mit bunten Bändern geschmückt werden, am Morgen des Ostermontags von Haus zu Haus und sammelten in großen Weidenkörben Eier. Bevor die gefüllten Körbe, die eigentlich Waschkörbe sind, am Marktplatz aufgestellt wurden, gab es mit ihnen einen musikalischen Festzug durch den Ort. Nachdem die insgesamt fünf Körbe vor dem Rathaus abgestellt waren, bildeten die beteiligten jungen Männer (in einem Alter von ca. 17 bis 24 Jahren) an den Seiten ein Spalier. Alle hielten ihre Birkenruten in der Hand. Ein Teil von ihnen trug ein Sweatshirt mit der Aufschrift „Traditioneller Eierlauf 1739 Remlingen". ${ }^{9}$ Hier wurden dann nach 14 Uhr der Eiersammler und der Läufer ausgelost.

Anschließend marschierte man zu den Klängen einer Blaskapelle und unter Begleitung der zahlreichen Zuschauer - es sollen 2-3000 gewesen sein - zu einer Wiese außerhalb des Ortes. Dort wurden 75 zumeist einfarbig gefärbte Ostereier in

\footnotetext{
${ }^{6}$ Nach MOSER (1957: 84)

${ }^{7}$ Nach Moser (1957: 84). Die Publikation von Karin GöBEL, Das Summenformelspiel. Zur Stellung eines Wettspieles zwischen Osterbrauch und Rechenbuchillustration, München 1987, in der auch das Eiersammeln und Laufen von Remlingen behandelt wird, stand mir leider bis zum Abschluß dieses Artikels nicht zur Verfügung.

${ }^{8}$ Für diesen Hinweis bedanke ich mich sehr herzlich bei meinem Kollegen Richard NEBEL (Bayreuth). Er war am Ostermontag des Jahres 1997 selbst vor Ort und teilte mir dann zahlreiche wichtige Informationen mit. Auf seine Angaben, auf die von ihm gemachten Fotos, die er mir dankenswerterweise zur Verfügung stellte, und auf einen in diesem Jahr erschienenen Zeitungsartikel (Anonym 1997a) beziehen sich die folgenden Ausführungen.

${ }^{9}$ Warum sich die Remlinger auf das Jahr 1739 beziehen, ist mir derzeit nicht bekannt. Eine gründliche historisch-ethnographische Untersuchung in Remlingen wäre sicherlich eine interessante und wichtige Forschungsaufgabe.
} 
einem Abstand von jeweils $75 \mathrm{~cm}$ in einer langen Reihe auf den Boden gelegt. Beim Wettkampf mußte dann der Eiersammler, der mit einem roten Band gekennzeichnet war, alle Eier einsammeln. Die Regel schrieb vor, daß alle immer einzeln aufgelesen und jeweils zum Ausgangspunkt zurückgebracht werden müssen. Der Anfang wurde mit dem weit entferntesten Ei gemacht. Zur gleichen Zeit mußte der Läufer, der in Remlingen auch als „Hase“ bezeichnet wird, eine genau festgelegte Strecke bis zum „Eierlaufstein“ (ca. 2,3 km) und zurück bewältigen. Wer zuerst seine Aufgabe erfüllt, ist Sieger. 1997 gewann der Eiersammler. Nach Aussagen von Einheimischen soll es aber meistens umgekehrt sein.

Auch für das leibliche Wohl war für die Teilnehmer und die vielen Besucher, die nicht nur aus Remlingen, sondern auch aus der Umgebung stammten, bestens gesorgt. Den ganzen Tag floß reichlich Bier, das an diesem Tag als „Eierbier” bezeichnet wird. $\mathrm{Zu}$ Essen gab es vor allem Bratwürste und Steaks. Den Abschluß dieses Dorffestes bildete am Abend eine große Tanzveranstaltung im Schützenhaus. 1997 hatte man auch eine Delegation aus Effingen in der Schweiz zu Gast, wo ebenfalls ein solcher Wettkampf - dort unter der Bezeichnung „Eierleset“ - durchgeführt wird. ${ }^{10}$

Für 1907 gibt es auch einen Beleg einer etwas anderen Variante des Eiersammelns aus Germete (bei Warburg) in Westfalen. Dort sammelten am Ostertag die Jungen zwei Stiege mit insgesamt 40 Eiern. Auf einem Gelände vor dem Dorf legte man diese in zwei Reihen mit je 20 Eiern nebeneinander auf den Rasen, jeweils zwei Schritt entfernt. Zwei Jugendliche wurden dann durch ein Los zum Eierlaufen bestimmt. Jeder der beiden, „Eierläufer“ genannt, erhielt einen Korb an den linken Arm gebunden. Dann mußte jeder so schnell er konnte eine Reihe Eier mit der rechten Hand aufheben und in den Korb legen. Wer zuerst seine Eier vollständig und unbeschädigt eingesammelt hatte, war Sieger. Er wurde mit einem roten Band geschmückt und hatte für den Rest des Tages freie Verpflegung. ${ }^{11}$ Ob sich diese oder eine ähnliche Tradition bis heute in dem Ort erhalten hat, war mir bis zur Beendi-gung dieses Artikels leider nicht bekannt.

Eine wahre theatralische Inszenierung war im südlichen Baden zumindest bis in die Mitte des 20. Jahrhunderts das „Eierspringen“ am Ostermontag. ${ }^{12}$ In Maskerade traten dort ein Eierspringer, zwei Wettfahrer, eine Hexe, zwei Hanswürste, ein Wachtmeister, ein Ortsdiener und ein Teufel auf. Für das Eierspringen, das von Jugendlichen vorbereitet und durchgeführt wurde, sammelte man zunächst 60 Eier. $\mathrm{Zu}$ Beginn des Spiels, das im Ortszentrum inszeniert wurde, traten zuerst alle maskierten Figuren auf und exerzierten eine zeitlang unter dem Kommando des Wachtmeisters. Dann legte einer der Jugendlichen die Eier in einem Abstand von etwa 30 $\mathrm{cm}$ an den Rand der Straße, die aus dem Ort führte.

Dann mußte der Eierspringer nacheinander alle 60 Eier in eine Wanne tragen, die am Anfang auf zwei Stühlen stand. Er mußte bei dem Ei anfangen, das am

\footnotetext{
${ }^{10} \mathrm{Zu}$ Ostereierspielen in der Schweiz, wo es ebenfalls eine reiche Tradition an solchen Spielen gibt, s. vor allem LIEBL (1957).

${ }^{11}$ SAUERMANN (1986: 358$)$

12 Nach DIETZE (1987: 69-70)
} 
weitesten vom Ausgangspunkt entfernt war. Während die Hexe die Aufgabe hatte, den Eierspringer zu stören, mußten die Hanswürste ihn verteidigen und die Hexe bestrafen. Zur gleichen Zeit fuhren die beiden Wettfahrer auf dem Fahrrad oder dem Motorrad eine bestimmte Strecke um die Wette. Der Verlierer mußte nach dem Spiel den teilnehmenden jungen Männern Wein spendieren. Sobald der erste Wettfahrer ans Ziel gelangt war, hörte der Eierspringer auf zu sammeln, gleichgültig, ob er fertig war oder nicht. Schließlich wurde noch die „böse Hexe“ vom Ortsdiener und den Hanswürsten gefangen genommen. Der Wachtmeister verlas laut die Streiche, die sie während des Spiels angestellt hatte. Dann wurde sie zum Tode verurteilt und „hingerichtet“. Der Teufel führte zusammen mit zwei Jungen die „erschossene“ Hexe auf einer Mistkarre „,in die Hölle“, womit das Spiel endete. Die 60 Eier des Spiels wurden nun für die Mitspieler zubereitet und verzehrt. Dies war der Auftakt für ein anschließendes Ortsfest mit Speis, Trank und Tanz.

Theatralische Inszenierungen bei religiösen Festen waren besonders in Süddeutschland früher weit verbreitet. Die Figur der Hexe und deren symbolische Hinrichtung ist eine makabre Erinnerung an die rigoros durchgeführten Hexenverfolgungen. Sie sind sicherlich ein Überbleibsel eines alten Glaubens, nach dem in der Osternacht die Hexen ihr Unwesen trieben und den Bauern Unglück brachten. Der Auftritt von Osterhexen ist heute vor allem in Schweden und Finnland bekannt. ${ }^{13}$ Dort wandern als Hexen verkleidete kleine Mädchen an Ostern von Haus zu Haus oder zu ihren Verwandten. Die Verkleidung besteht aus Kopftüchern oder Hüten, schwarzgemalten Augenbrauen, Sommersprossen und rotgemalten Lippen, alten Röcken, alten Schuhen und bunten Federn. Sie haben ein Bündel bunt geschmückter Weidenruten in der Hand, mit denen sie die Bewohner berühren und ihnen Glück für das kommende Jahr wünschen. In Schweden und Finnland gelten die Osterhexen heute als augesprochene Glücksbringer.

Im hessischen Dorf Külte, das heute zu Volkmarsen im Waldecker Land gehört, ist das Eiersammeln bis zur Gegenwart einer der Höhepunkte des Jahreskreislaufes. 1997 fand es dort zum 144. Mal statt - man kann also davon ausgehen, daß es mindestens seit 1854 durchgeführt wird. Wenn es einige Male nicht stattgefunden hat, ist es auch noch älter. Leider liegen mir über die Geschichte des Spiels in diesem Dorf keine näheren Informationen vor, so daß ich mich bei den folgenden Ausführungen nur auf die letzten Jahre beziehen kann. ${ }^{14}$

Träger und Organisator ist der Burschenclub Külte, der traditionell für die $\mathrm{Ge}-$ staltung dieses Dorffestes zuständig ist. Nur wer nicht verheiratet und verlobt und zudem über 18 Jahre alt ist, kann darin aufgenommen werden. Das ganze Jahr über pflegt man Geselligkeit mit Ausflugsfahrten und gemütlichen Zusammenkünften. Jeweils am Palmsonntag ziehen die Mitglieder dann laut gackernd von Haus zu Haus durch den Ort, um Eierspenden für das Spiel zu sammeln. Unter der Führung

\footnotetext{
${ }^{13}$ S. hierzu z. B. ESKERÖD (1953) und SIPPEL (1995). Für interessante Informationen zum Thema Osterhexen bedanke ich mich sehr herzlich bei Kaarina DEHLS (Hamburg), Auli Kaarina KosKISTEFFEN (Gifhorn) und Marianne SinEMUS-AMMERMANN (Tangstedt).

${ }^{14}$ Anonym 1991 und 1997b. Für die beiden Artikel und ihre ergänzenden Informationen bedanke ich mich sehr herzlich bei Gerda und Heinz RAMBOw (Bad Arolsen).
} 
des jeweiligen Vorsitzenden wurden in den letzten Jahren stets über 1500 Hühnereier in einer „Kiepe“ genannten Handkarre bei den Bewohnern zusammengetragen. Wer keine Eier spendiert oder die Haustüre nicht öffnet, bekommt als Zeichen der Ablehnung Sägemehl vor das Haus gestreut. Für die Mühe des Eiersammelns, das gewöhnlich einen ganzen Tag in Anspruch nimmt, belohnen die Einwohner die jungen Männer oft mit einem Schnäpschen. Durchhaltevermögen ist hierbei gefragt, da der Einsatz sehr lange dauert und die zerbrechliche Fracht auch noch sicher zum Lager transportiert werden muß.

Die Verwendung der Eier war dann in den Jahren 1991 und 1997, aus denen mir nähere Angaben vorliegen, unterschiedlich. 1991 wurden die „Rohlinge“ bis Karfreitag gelagert und dann 100 Stück für den Wettkampf hart gekocht und bunt bemalt. Die übrigen Eier wurden noch vor dem Ostersonntag verkauft - der Erlös diente der Aufstockung der Clubkasse. 1997 lud der Burschenclub dagegen am Gründonnerstag vor dem Feuerwehrhaus zu einem großen Eierbacken ein. Am Karfreitag wird der Burschenclub ab $20 \mathrm{Uhr}$ auch von den Mädchen des Dorfes beim Eierfärben für das große Spiel, bei dem jedes Jahr zahlreiche Besucher aus der ganzen Umgebung erwartet werden, unterstützt.

Der Höhepunkt findet dann am Ostersonntag ab 14 Uhr statt. Die Eier - in den letzten Jahren waren es zwischen 80 und 100 - werden auf Sägemehl entlang der Straßenmitte ausgelegt. Unter der strengen Aufsicht eines Clowns begeben sich zwei Eierleser auf die Bahn und sammeln die Eier Stück für Stück auf. Sie müssen sie in einen Korb legen, der von einer „Glucke“ behütet wurde. Zur Belustigung der Zuschauer werden die beiden auch immer wieder von dem Clown angetrieben. Zur gleichen Zeit versuchen zwei Läufer eine genau festgelegte Strecke abzulaufen. Die Gruppe, die ihre Aufgabe zuerst erfüllt hat, ist Sieger. Die hartgekochten „Leseeier“ werden nach der Siegerehrung vom Burschenclub an die jüngeren Zuschauer verteilt. Die ganze Aufführung endet mit einem fröhlichen Dorffest mit Musik und Tanz bis spät in die Nacht hinein.

\section{IN DEUTSCHLAND ALLGEMEIN BEKANNTE UND VERBREITETE OSTEREIERSPIELE}

Die im folgenden genannten Spiele werden praktisch ausschließlich von Kindern ausgeübt. Alle diese Spiele haben unzählige individuelle Varianten, die gar nicht alle aufgezählt werden können. Es handelt sich daher hierbei nur um einen kursorischen Überblick.

Das wohl gebräuchlichste Spiel - obwohl es meistens gar nicht mehr als solches bezeichnet wird - ist das Suchen von Ostereiern. Zumeist wird es in den Familien praktiziert, indem an den Osterfeiertagen die Eier von den Eltern oder von den älteren Geschwistern in der Wohnung - oder bei schönem Wetter im Freien - versteckt werden. Die Kleinen dürfen dann auf Entdeckungsreise gehen und die gefundenen Schätze, wie Eier, Osterhasen oder ganze Nester in Besitz nehmen. Gerne wird da- 
bei auch auf ein Punktesystem zurückgegriffen, bei dem man vorher den Farben der einzelnen Eier Punkte zuweist. Der Sieger wird dann nicht nach der Anzahl der gefundenen Eier, sondern nach der Punktezahl ermittelt. ${ }^{15}$

Besonders beliebt ist das Ostereiersuchen auch im Kindergarten. So empfiehlt z. B. Rolf KRENZER (1984) folgendes Spiel: Einer spielt den Osterhasen. Dieser versteckt für jeden Mitspieler ein Osterei im Zimmer, auf dem Spielplatz oder im Garten. Jeder darf dann ein Ei suchen. Wer zuerst ein Ei gefunden hat, darf beim nächsten Spiel der Osterhase sein. Eine andere Möglichkeit ist, daß jeder ein Ei in einer bestimmten Farbe auswählt. Es darf dann nur dieses Ei und nicht die anderen gesucht werden. Entdeckt jemand ein anderes Ei, soll er es den übrigen Mitspielern nicht verraten. Zusätzliche Hilfe kann beim Suchen durch die Hinweise „kalt“ oder „heiß“" gegeben werden. ${ }^{16}$

Ebenfalls weitbekannt ist das Eierlaufen - eine wesentlich einfachere Form, als das weiter oben ausgeführte Eiersammeln und -laufen. Hier gibt es die unterschiedlichsten Varianten, ein Osterei in Wettkampfform zu einem bestimmten Ziel zu tragen. ${ }^{17}$ Das Osterei kann von den Kindern in einem Eßlöffel, mit beiden Händen, in einer Hand oder in einem Eierbecher getragen werden. Sieger ist, wer sein Ei nicht verliert und als erster ans Ziel gelangt. Das Spiel kann auch mit Hindernissen gestaltet werden, z. B. unter einer Stange hindurch, über einen Hügel hinweg oder über einen Gartenzaun. Für die Größeren können auch bestimmte „Pflichtfiguren“, wie Kniebeugen machen, sich drehen oder rückwärts gehen, eingeführt werden. In Hamburg wurde 1997 beim 13. Norddeutschen Ostermarkt im Museum für Völkerkunde am Palmsonntag ein Eierlauf für Kinder unterschiedlichster Altersklassen veranstaltet. Dieser fand nicht nur einen riesigen Zulauf bei Kindern, sondern sorgte auch für eine begeisterte Stimmung bei den Eltern, die unermüdlich ihre Kinder anfeuerten. Dieses ursprüngliche Ostereierspiel hat sich so verbreitet, daß es zu einem allgemeinen Kinderspiel wurde und auch vielfach während des Jahres mit einfachen Eiern, Äpfeln oder gar Tennisbällen durchgeführt wird. ${ }^{18}$

Auch das Eierrollen oder Eierzielen ist in Deutschland weithin verbreitet und wird in zahlreichen Variationen praktiziert. Eine der einfachsten Formen ist das Hinunterrollen an einem Abhang, wobei der gewinnt, dessen Ei am weitesten gerollt oder am wenigsten lädiert ist. Man kann auch eine Rollbahn herstellen, indem man z. B. ein breites Brett an einem Ende auf Backsteine oder Holzklötze legt.

Brigitte BLOBEL (1980) empfiehlt, zwei Besenstiele an den Enden so zusammenzubinden, daß in der Mitte eine Schräge entsteht. Diese Laufschiene wird dann gegen einen Tisch, einen Stuhl oder eine Astgabel gelegt und so in Schräglage fixiert. Jeder Spieler erhält drei hartgekochte Eier in seiner Spielfarbe, rot, blau, gelb, grün - es können sich beliebig viele Spieler beteiligen. Nacheinander läßt jeder sein Ei in der Laufrinne hinunterrollen. Je mehr Eier im Spiel sind, desto häufiger

\footnotetext{
${ }^{15}$ S. hierzu z. B. KRUHÖFFER-MIELKE (1981: 26)

${ }^{16}$ KRENZER (1984: 48-49)

${ }^{17}$ S. z. B. KRUHÖFFER-MieLKe (1981: 26); MeHLing (1984: 132); KRENZER (1984: 48); Das fröhliche Osterfest (1985: 37); DIETZE (1987: 70); LEISKA (1988: 31)

${ }^{18}$ Vgl. GÖÖCK (1988: 359)
} 
kann es passieren, daß ein Ei auf ein anderes trifft. Sieger ist, wer am Ende die größte Anzahl unbeschädigter Eier besitzt. ${ }^{19}$

Die Eier können auch über den Rasen um die Wette gerollt werden. Wer am weitesten mit seinem Ei kommt, ist Sieger und darf bei der nächsten Runde als erster beginnen. Rolf KRENZER (1984) führt für den Kindergarten folgende weitere Möglichkeiten an: Ein Osterei wird mit den Händen um die Wette zum Ziel gerollt. Die Kinder sitzen in der Hocke und versuchen, das Osterei um sich herum zu rollen. Oder man markiert auf dem Boden einen Kreis und jeder muß versuchen, ein oder mehrere Eier in den Kreis zu rollen. Es wird vorher vereinbart, wie nahe die Mitspieler an den Kreis herantreten dürfen. Und schließlich kann man die Ostereier auch in eine Grube rollen lassen. ${ }^{20}$

Das letzte Spiel wird auch als „Eierzielen“ bezeichnet und wird gerne von größeren Kindern gespielt. Man braucht hierfür eine Grube oder ein Loch, wo ein Ei liegen bleiben kann. Von den einzelnen Wettkämpfern werden die Ostereier dann aus einiger Entfernung auf das Loch zugerollt. Wer es fertigbringt, das Ei direkt in der Grube zu versenken, hat die übrigen Eier, die ihr Ziel nicht erreicht haben, gewonnen. Man kann auch in einer sehr großen Entfernung beginnen und jeweils derjenige, der dem Ziel am nächsten liegt, darf weiterspielen. Wessen Ei zuerst im Loch ist, hat gewonnen. ${ }^{21}$

Dieses Kullern von Ostereiern hat eine besondere Ausprägung und Tradition bei den Sorben, unter der Bezeichnung Waleien (walkowanje) erfahren. Ursprünglich soll ihm ein Fruchtbarkeitszauber zugrunde liegen, bei dem man glaubte, durch das Rollen von Eiern über die Wiesen und die Felder im Frühling das Wachstum der Saaten besonders günstig beeinflussen zu können. ${ }^{22}$ In den meisten Orten wird eine kleine Grube mit abschüssiger Bahn ausgehoben, die bis 1,5 Meter breit und bis drei Meter lang ist und am Ende des Gefälles eine Tiefe von ca. 30 bis $40 \mathrm{~cm}$ hat. In verschiedenen Gegenden der Niederlausitz findet sich noch eine weitere Form der Waleie. Bei dieser errichtet man eine Sandaufschüttung mit einer schiefen Ebene, auf der, ebenfalls aus Sand, ornamentartige Hindernisse angebracht werden.

Eine beliebte Spielregel ist dann folgende: Der erste Spieler legt ein Ei in die Grube. Der nachfolgende Spieler muß versuchen, mit seinem in die Grube rollenden Ei das erste zu treffen. Verfehlt er es, bleibt auch sein Ei dort liegen und der nächste Mitspieler ist an der Reihe. Trifft er das Ei, darf er es behalten und sein Ei wieder herausnehmen oder er bekommt ein Geldstück oder eine Süßigkeit. Außerdem darf er das Spiel so lange fortsetzen, bis er keinen Erfolg mehr hat. Da nicht alle Mitspieler treffen, sammeln sich oft mehrere Eier in der Grube an. Trifft ein Spieler mehrere Eier zugleich, wird er hierfür entsprechend honoriert. ${ }^{23}$

\footnotetext{
19 BLOBEL (1980: 86)

${ }^{20}$ KRENZER (1984: 47-48)

21 S. hierzu z. B. MEHLing (1984: 131); Das fröhliche Osterfest (1985: 47)

22 Nach SCHMIDT (1980: 40)

23 SCHMIDT (1980: 40-44)
} 
In Deutschland allgemein beliebt ist das Eierticken, Eierstoßen oder Eierknicken. Dabei stoßen zwei Spieler die Eier mit den stumpfen oder den spitzen Enden gegeneinander. Man kann die Regel aufstellen, daß erst die spitzen und dann die stumpfen Seiten zum Einsatz kommen. Gewonnen hat der Besitzer des am wenigsten lädierten Eies. Er erhält als Prämie das unterlegene Ei. In der einschlägigen Literatur wird auch vor Falschspielern gewarnt, die mit besonders präparierten oder gar Gipseiern antreten. ${ }^{24}$

Beim Eierblasen wird ein hohles Ei in die Mitte eines Tisches gesetzt. Alle Spieler setzen sich dicht an den Tisch und legen ihre Arme an den Tischrand, um so eine Absperrung für das Ei zu bilden. Dann beginnt der erste Spieler das Ei anzublasen, das nun auf einen Mitspieler zurollt. Dieser muß dagegenblasen, damit ihn das Ei nicht berührt. Wer von dem Ei getroffen wird, erhält einen Punkt. Wer am Ende die wenigsten Punkte auf seinem Konto hat, ist der Sieger - derjenige, der am meisten hat, ist der Verlierer. Bei diesem Spiel kommt es auf gute Reaktionen und auf ein kräftiges Blasvermögen an..$^{25}$

Eine recht einfache Form ist das Eierfangen. Hierbei werfen sich zwei oder mehr Kinder gegenseitig Ostereier zu. Wenn einer der Mitspieler das Ei nicht auffängt, so gehört es demjenigen, der geworfen hat. Verschiedene Erschwernisse können den Spaß erhöhen, etwa wenn alle Teilnehmer nur auf einem Bein stehen, sich niederknien oder nur mit einer Hand fangen dürfen. ${ }^{26}$

\section{MODERNE SONDERFORMEN VON OSTEREIERSPIELEN}

Die folgenden Ostereierspiele sind Empfehlungen in verschiedenen Spiele- und Bastelbüchern für die Osterzeit. Sie kommen sicherlich nur in Einzelfällen zum Einsatz und haben sich bisher nicht allgemein ausgebreitet.

Walter SPERLING (1981) beschreibt ein Eiersuchspiel, das auf einem Tisch durchgeführt wird. Dazu müssen vier große und ineinander befindliche Kreise mit einem Zirkel auf Papier gezeichnet werden. Diese werden mit fünf geraden Linien in Sektoren aufgeteilt. In den Mittelkreis kann man zur Dekoration einen Hasen malen. Das Spielzubehör besteht zudem noch aus zwei mal drei Eiern, z. B. drei rote und drei gelbe. Diese werden ebenfalls auf Papier gemalt und ausgeschnitten. Die beiden Spieler erhalten jeder drei gleichfarbige Eier, die sie jeweils auf ihrer Spielseite in die drei Fächer der horizontalen Leiste legen. Außerdem benötigt man einen Würfelbecher mit einem Würfel. Nach der Auslosung des Spielanfängers wird abwechselnd gewürfelt. Nach jedem Wurf werden die Eier entsprechend der geworfenen Augenzahl in Bewegung gesetzt. Ziel ist die den Spielern jeweils gegenüberliegende Seite. Wer zuerst mit seinen Eiern die gegnerischen drei Felder besetzen kann, ist Sieger. Dabei muß man mit den Eiern immer im Kreise herum spielen und

\footnotetext{
24 S. z. B. BLOBEL (1980: 36); Das fröhliche Osterfest (1985: 36).

25 BLOBEL (1980: 86); Das fröhliche Osterfest (1985: 37); LEISKA (1988: 32).

26 Mehling (1984: 131); Das fröhliche Osterfest (1985: 37).
} 
es darf kein gegnerisches Ei aus seinem Nest oder aus einem anderen Feld hinausgeworfen werden, wenn man beim Auszählen des Wurfes dort hingelangt. Das im Lauf befindliche Ei muß hinter das gegnerische gestellt werden, wenn die Eier beim Würfeln aufeinandertreffen sollten. Der Spielverlauf ist durch überlegtes Ziehen und geschicktes Taktieren zu beeinflussen. ${ }^{27}$

Ein Spiel, das ebenfalls erst gebastelt werden muß, ist „Versteckte Eier - Ein Spiel für kreative Detektive“, das von Christina PFEIFFER und Renate VoGL (1994) empfohlen wird. Hierzu werden zwei Eierkartons ohne Deckel an den Längsseiten zusammengeklebt und bemalt. Die Seitenwände werden ausgeschnitten und rechtwinklig an zwei Seiten des Kartons nach obenstehend geklebt. In einer Reihe werden über die Eierfächer Zahlen und in der anderen Buchstaben geschrieben. So erhält jedes Eierfach ähnlich wie beim Schachspiel Buchstaben/Zahlen-Bezeichnungen (hier z. B. A1, A2, usw., B2, C3, D4, usw.). Dann werden acht Eier beispielsweise gelb und zwei pink angemalt. Jeweils acht und zwei müssen die selbe Farbe bekommen. Die Spielregeln sind folgendermaßen: Zwei Spieler versuchen, die Verstecke des anderen zu finden. Jeder versteckt vier gelbe Eier und ein pinkfarbenes Ei. Der erste Spieler nennt nun eine Zahl und einen Buchstaben, wie sie auf der Kartonwand stehen. Ist auf dem Kreuzungspunkt dieser Angaben beim anderen Spieler ein Ei versteckt, muß dieser es abgeben. Wer ein pinkfarbenes Ei findet, erhält ein verlorenes wieder zurück und ist gleich noch einmal an der Reihe. ${ }^{28}$

Viele einfallsreiche Ostereierspiele, die zum größten Teil alte und traditionelle Vorbilder haben, finden sich in dem neuen Buch von Renate DöRNER (1997). Beim Eierkettenspiel können sich viele Kinder und auch Erwachsene beteiligen. Gleichzeitig entsteht bei diesem Spiel ein dekorativer Osterschmuck für das Haus oder den Garten. An Materialien werden für jede Gruppe zwei bis vier Eier pro Person, Plakatfarben, Pinsel und eine lange Schnur benötigt. Ziel des Spiels ist es, in Gemeinschaftsarbeit eine Eierkette von großer Länge herzustellen, die dann Osterbaum, Treppenhaus, Balkon oder Garten schmücken kann.

Dazu werden zunächst zwei gleich große Gruppen gebildet. Beide Gruppen erhalten eine vorher festgelegte Anzahl von rohen, ungefärbten Eiern. Man kann auch noch Bänder oder getrocknete Blumen als Material für die Dekoration mit hinzunehmen. Die Aufgabe besteht darin, in möglichst kurzer Zeit die Eier vorsichtig in eine große Schüssel auszublasen, zu bemalen und aufzufädeln. Ob dies in Arbeitsteilung erfolgt oder jeder die Eier allein ausbläst und bemalt, entscheidet jede Gruppe selbst. Sieger ist die Gruppe, die zuerst fertig ist und deren Eierkette am längsten ist. Für jedes Ei, das bei der Bearbeitung kaputtgeht, werden allerdings zwei Minuten abgezogen. Am Ende werden die beiden Eierketten zusammengebunden und die Siegergruppe darf entscheiden, wo die Kette aufgehängt wird. Anschließend wird aus dem Inneren der vielen Eier Kuchen gebacken. ${ }^{29}$

\footnotetext{
${ }^{27}$ SPERLING (1981: 66-68). Das selbe Spiel ist in Kurzform auch bei DIETZE (1987: 72) beschrieben.

${ }^{28}$ PFeifFER-VOGL (1994: 30)

${ }^{29}$ DÖRNER (1997: 109)
} 
Beim Eierkricket werden etwa $40 \mathrm{~cm}$ lange Weidenruten als Bögen ins Gras gesteckt. Bei der Anordnung der Bögen muß darauf geachtet werden, daß die Spieler ungehindert ihre Schläge ausführen können. Die Spieler stoßen mit der schmalen Seite eines Schrubbers das Ei durch die Bögen. Wer zuerst mit den wenigsten Schlägen wieder beim Start ankommt, ist Sieger. Ein Spielleiter notiert die Schläge. Bei schlechtem Wetter kann das Kricketspiel auch im Haus zwischen Stuhlbeinen und Hockern stattfinden. ${ }^{30}$

Für die Eierrutsche benötigt man etwa 20 Plastikbecher, doppelseitiges Klebeband, Wellpappreste, Zweige mit Verästelungen, Schere, Küchenmesser, Klebstoff und Papierreste. In den unteren Teil der Becher wird mit dem Messer ein Loch gestochen und dann mit der Schere der Becherboden abgeschnitten. An den unteren Becherrand wird ein Streifen doppelseitiges Klebeband befestigt. Die Schutzfolie wird vom Klebeband abgezogen und die Becher mit den Klebstreifen nach unten zusammengesteckt. Zusätzlich gibt man noch einige Tropfen Klebstoff zwischen die Becherwände und läßt ihn trocknen. Verlängerungsröhren aus einem etwa $15 \mathrm{~cm}$ langen Wellpappestück können die Kinder zusätzlich in den oberen und den unteren Becher stecken. Bunte Papierschnipsel oder -streifen werden zur Verzierung um die Becher geklebt.

Die Becherröhre benötigt ein steiles Gefälle. Dazu werden aus Sträuchern Verästelungen heraussortiert und in unterschiedlichen Längen abgebrochen. Die längste Astgabel sollte z. B. 60 bis $70 \mathrm{~cm}$ und die kürzeste $15 \mathrm{~cm}$ lang sein. Diese Astgabeln werden in steilen Abstufungen in den Rasen gesteckt. Wenn die Becherröhre darauf gelagert ist, können die ersten Rutschversuche unternommen werden. Wichtig ist, daß die Röhre in der Mitte nicht zu stark durchhängt, sonst bleiben die Eier darin hängen. Mit etwas Geschick entsteht sogar eine Rutsche mit Kurve. Anstelle der Astgabeln eignen sich auch Schachteln, Hocker und Kisten. ${ }^{31}$

Bei diesen modernen Spielvorschlägen für Ostern steht eindeutig das Basteln und die Kreativität im Vordergrund. Sie dürften für Kinder, Eltern und Pädagogen eine willkommene Abwechslung sein. Für eine weiträumige Verbreitung und in Hinblick auf das Entstehen einer neuen Tradition haben sie aber wohl kaum eine Chance.

\section{LITERATUR}

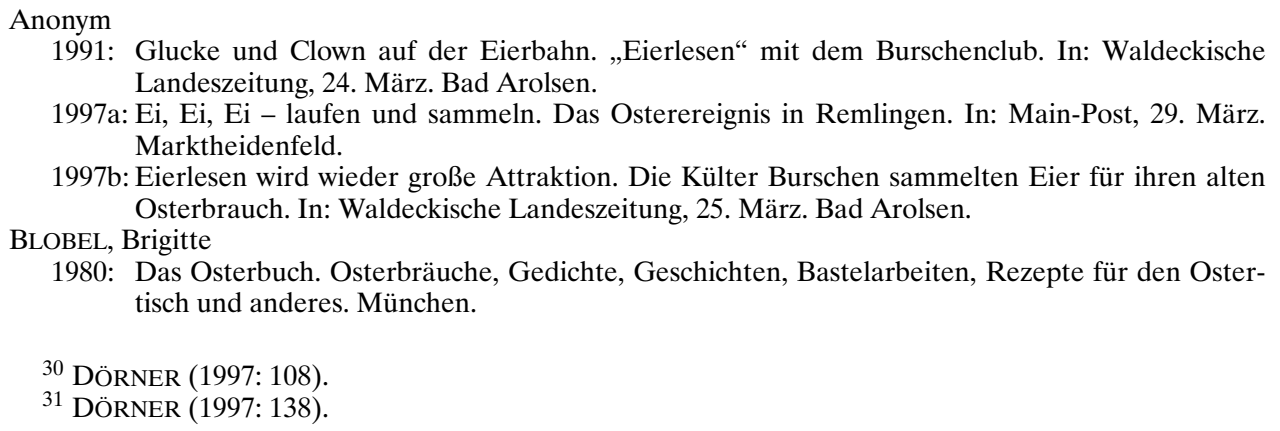

1997b: Eierlesen wird wieder große Attraktion. Die Külter Burschen sammelten Eier für ihren alten Osterbrauch. In: Waldeckische Landeszeitung, 25. März. Bad Arolsen.

1980: Das Osterbuch. Osterbräuche, Gedichte, Geschichten, Bastelarbeiten, Rezepte für den Ostertisch und anderes. München.

30 DÖRNER (1997: 108).

31 DÖRNER (1997: 138). 
DIETZE, Katharina

1987: Oster(eier)spiele. In: Rüdiger Vossen et al., Ostereier - Osterbräuche (Wegweiser zur Völkerkunde, 25). 4. Auflage. Hamburg.

DÖRNER, Renate

1997: Kunterbunte Osterzeit. Das Spiel-, Spaß- und Bastelbuch für die ganze Familie. Augsburg.

ESKERÖD, Albert

1953: Aarets fester. Stockholm.

Das fröhliche Osterfest

1985: Das fröhliche Osterfest. Hamburg.

GÖÖCK, Roland von

1988: Das große Buch der Spiele. 1000 Spiele für drinnen und draußen. München.

KRENZER, Rolf

1984: Die Osterzeit im Kindergarten. Texte, Lieder, Spiele, Bastelvorschläge. Lahr.

KRUHÖFFER, Barbara-MIELKE, Heinz-Peter

1981: Osterfest und Osterbrauch (Schriften des Mindener Museums für Geschichte, Landes- und Volkskunde. Volkskundliche Reihe, 1). Lübeck.

LEISKA, Hildegard

1988: Wir feiern Ostern. Bastelanregungen, Spiele und Brauchtum. München.

LIEBL, Elsbeth

1957: Ostereierspiele im Atlas der schweizerischen Volkskunde. In: Schweizerisches Archiv für Volkskunde, 53, S. 61-67. Basel.

MEHLING, Marianne

1984: Rund ums Osterfest. Geschichten, Gedichte, Lieder - Bräuche, Rezepte und Basteleien. München.

MOSER, Hans

1957: Osterei und Ostergebäck. Brauchgeschichtliches aus bayerischen Quellen. In: Bayerisches Jahrbuch für Volkskunde, 1957, S. 67-89. Regensburg.

PFEIFFER, Christina-VOGL, Renate

1994: Das große Osterbastelbuch für die Familie. Augsburg.

SAUERMANN, Dietmar (ed.)

1986: Ostern in Westfalen. Materialien zur Geschichte eines volkstümlichen Kirchenfestes (Beiträge zur Volkskultur in Nordwestdeutschland, 46). Münster.

SCHMIDT, Ernst

1980: Bunte sorbische Ostereier. Bautzen.

SIPPL, Elsa

1995: Ostern in Finnland. In: Rengas, 4/95.

SPERLING, Walter

1981: Werken und Spielen zu Ostern. Stuttgart. 